\title{
Revision, uptake and coding issues related to the open access Orchard Sports Injury Classification System (OSICS) versions 8, 9 and I0.I
}

This article was published in the following Dove Press journal:

Open Access Journal of Sports Medicine

9 October 2010

Number of times this article has been viewed

\section{John Orchard' \\ Katherine Rae' \\ John Brooks ${ }^{2}$ \\ Martin Hägglund ${ }^{3}$ \\ Lluis Til ${ }^{4}$ \\ David Wales ${ }^{5}$ \\ Tim Wood ${ }^{6}$}

'Sports Medicine at Sydney University, Sydney NSW Australia; ${ }^{2}$ Rugby Football Union, Twickenham, England, UK; ${ }^{3}$ Department of Medical and Health Sciences, Linköping University, Linköping, Sweden; ${ }^{4}$ FC Barcelona, Barcelona, Catalonia, Spain; ${ }^{5}$ Arsenal FC, Highbury, England, UK; ${ }^{6}$ Tennis Australia, Melbourne, Vic, Australia
Correspondence: John Orchard Sports Medicine at Sydney University, Cnr Western Ave and Physics Rd, University of Sydney NSW, Australia 2006

$\mathrm{Tel}+61293518118$

Fax +6I 293518123

Email johnworchard@gmail.com

\begin{abstract}
The Orchard Sports Injury Classification System (OSICS) is one of the world's most commonly used systems for coding injury diagnoses in sports injury surveillance systems. Its major strengths are that it has wide usage, has codes specific to sports medicine and that it is free to use. Literature searches and stakeholder consultations were made to assess the uptake of OSICS and to develop new versions. OSICS was commonly used in the sports of football (soccer), Australian football, rugby union, cricket and tennis. It is referenced in international papers in three sports and used in four commercially available computerised injury management systems. Suggested injury categories for the major sports are presented. New versions OSICS 9 (three digit codes) and OSICS 10.1 (four digit codes) are presented. OSICS is a potentially helpful component of a comprehensive sports injury surveillance system, but many other components are required. Choices made in developing these components should ideally be agreed upon by groups of researchers in consensus statements.
\end{abstract}

Keywords: sports injury classification, epidemiology, surveillance, coding

\section{Introduction}

Ongoing sports injury surveillance is a fundamental pillar of sports injury prevention, ${ }^{1-4}$ in a process described originally by van Mechelen et $\mathrm{al}^{5}$ and more recently by Finch. ${ }^{6}$ It is to sports medicine what cancer registries are to oncology or traffic accident databases are to traumatology. One of the reasons why sports injury prevention has been generally elusive is that there are very few long-standing sports injury surveillance systems in the world. Many of the existing systems are funded by professional sporting competitions, as part of due diligence, but on the premise that the raison d'être for professional sport is entertainment rather than injury containment. Some of the longstanding injury surveillance systems in non-professional sport have led to successful injury prevention, such as the Accident Compensation Corporation (ACC) in New Zealand $^{7-9}$ and the National Register of Catastrophic Spinal Injuries in the USA, ${ }^{10}$ plus the introduction of breakaway bases in some amateur baseball leagues. ${ }^{11}$ In professional sport there have been some successful reductions of specific injuries. ${ }^{12}$

An important part of injury surveillance is coding of injury diagnoses, although it is important to note that there are many other aspects to injury surveillance systems than just coding. ${ }^{1}$ The two major purposes of coding are to facilitate retrieval of records of a certain type for future analysis and to collate diagnoses into common groups to follow trends in injury incidence and prevalence ${ }^{13}$ (as per the van Mechelen paradigm ${ }^{5}$ ). There is a trade-off between simplicity (which assists ease of use and categorization) and a comprehensive list of codes (which improves accuracy). ${ }^{13,14}$ 
Only a few papers have analysed issues related to coding of sports medicine diagnoses, such as accuracy. ${ }^{14,15}$ These issues have some importance however, as they have the ability to affect the comparability and compliance of injury surveillance.

The Orchard Sports Injury Classification System (OSICS) $)^{13,14,16,17}$ is perhaps the world's most widely used injury coding system in sports medicine. Its uptake is due to a combination of being a free-to-use system and having codes which are specific to sports medicine.

OSICS was updated to version 6 in 1998, version 7 in 2000 and version 8 in 2002. In 2005, it was determined that, for some purposes, there were permanent inadequacies in a three character coding system. ${ }^{14}$ Therefore, a four character system was created in 2007. ${ }^{13}$ It was named OSICS version 10 as it was envisaged that there would still be a role for a three character system and that when OSICS 8 was updated it should be called OSICS 9. Minor updates which only change a few codes can be made, with versions progressing using a decimal point (for example this study will include OSICS version 10.1 which is only marginally different to OSICS 10).

The aims of this study were to:

1. document the extent of uptake of OSICS by major research groups in sports medicine

2. discuss the issues and problems with implementation and coding of OSICS from experienced stakeholders, along with suggestions for how to handle conflicts

3. present suggested standard injury categories for some of the world's major sports where injury classification is used, and

4. present updated versions of OSICS (version 9 and version 10.1)

\section{Methods}

The first author and creator of OSICS (John Orchard) along with the primary creator of OSICS version 10 (Katherine $\mathrm{Rae}$ ) are in regular email contact with key stakeholders in the sports of football (soccer), Australian football, cricket, tennis and rugby union, the sports in which OSICS has primarily been used. The majority of these stakeholders are included as coauthors in this paper. In addition, programmers associated with various athlete management systems, who have installed OSICS as a freeware add-on, were consulted via email. This consultation process was fairly informal, particularly by comparison to the processes involved in updating more comprehensive and established systems like the International
Classification of Diseases (ICD). However, this consultation process was the same as the process for updating OSICS versions 1 through 7 .

To further assess uptake of OSICS, various literature searches were performed for the expressions 'OSICS' and 'Orchard codes' (PubMed, Sport Discus, Google Scholar) as well as a citation tracker for the specific OSICS papers. ${ }^{13,14,16,17}$

\section{Results}

The following groups/sports have been identified as users of OSICS:

1. Australian Football League injury surveillance, which has used OSICS for 18 seasons and was the original system for which the OSICS codes were designed. ${ }^{12,18-22}$ OSICS has also been used for other Australian football studies. $^{23}$

2. Cricket Australia injury surveillance system ${ }^{24,25}$ and international consensus definitions for cricket injuries. ${ }^{3,4}$

3. UEFA injury surveillance system ${ }^{26-28}$ and international consensus definitions for football (soccer) injuries. ${ }^{1}$

4. A rugby union injury surveillance instrument, ${ }^{29}$ an international consensus statement on injury definitions and data collection for rugby union injuries ${ }^{2}$ and the England Rugby Injury and Training Audit which has used OSICS since its inception in 2002. ${ }^{30,31}$

5. The international consensus definitions for tennis s2,33 $^{3}$ and Tennis Australia injury surveillance system.

6. Experts providing advice to the International Olympic Committee on injury surveillance in team sports. ${ }^{34}$

7. Multiple athlete management systems including Sports Injury Manager, Athletic Logic, Fairplay and Injury Tracker.

OSICS version 8 has been previously identified as being superior to the ICD 10 for coding sports injury diagnoses. ${ }^{14}$ However, deficiencies in OSICS 8 led to the development of a new version of OSICS. This was labelled version 10 , as it included a significant modification of the inclusion of a fourth character. ${ }^{13}$ This enabled OSICS 8 to be updated at a later date to a version 9, still using three digits only, which is presented in this paper. OSICS 10 , by virtue of a greater number of codes, is able to give more comprehensive diagnostic differentiation and hence greater diagnostic accuracy than a 3 digit system. ${ }^{13-15}$ The three digit system still retains a potential advantage of having fewer choices for the user and therefore finding an applicable code from a shorter list may be easier. However, this advantage is 
obviously negated if the user feels that no code is correct or specific enough.

In a computerised system, it is not ideal to have an unfiltered drop down list of all OSICS codes to choose between. This involves many hundreds of codes, often making it hard for the user to find the most applicable code (particularly in OSICS version 10). It is recommended that programmers include filters for any or all of body part, injury type, or keywords, which can then reduce a dropdown list of potential codes to a small number from which to choose. An 'intelligent' system could use keywords from a text diagnosis provided to suggest the best fit OSICS codes to form a drop down list, from which the user could choose the most appropriate code. An even more intelligent system could allow the user to expand the drop down list (if nothing suitable was suggested in the drop down list) or narrow it (if too many codes were provided to choose between). If no filtering is used, it would be recommended that the body part was chosen as the first word of the text descriptor field, which would help organise an alphabetical list of text fields (eg, calcaneus fracture, rather than fractured calcaneus).

Suggested or example injury categories for some of the major sports which use OSICS are presented in Tables 1, 2 and 3. These vary between sports to reflect the relevance of various diagnoses. For example, abdominal ('side') strains are common injuries in fast bowlers in cricket, but rare injuries in most other sports. Stingers or burners (cervical nerve root compression injuries) are common injuries in rugby union but again uncommon in other sports. These injury categories should probably be further refined by consensus groups within the sport, hence are listed as suggested rather than recommended at this stage. Some injury categories need to be separated depending on the injury definition in a sport. For example, in the football codes, head and facial lacerations are common enough to warrant a separate injury category if the injury definition is based on medical treatment. However, if the injury definition is based on time loss (with a threshold of 24 hours or more), then head and facial lacerations probably do not warrant their own separate category, as they rarely result in time loss other than possibly on the day of occurrence. The suggested and example categories represent the bias of the primary author and are not necessarily the preferences of the other authors in their particular sports. They have been included because of the presence of injury category code numbers for OSICS 9 in the Appendix.
Table I Suggested global injuries category for merging of OSICS codes

\begin{tabular}{|c|c|c|c|}
\hline Region ID & Region & Injcat ID & Injury category \\
\hline \multirow[t]{10}{*}{1} & Head and neck & 1 & $\begin{array}{l}\text { Head and neck soft } \\
\text { tissue trauma }\end{array}$ \\
\hline & & 3 & Eye injuries \\
\hline & & 5 & Concussion \\
\hline & & 6 & Facial bone fractures \\
\hline & & 9 & $\begin{array}{l}\text { Other head organ } \\
\text { damage }\end{array}$ \\
\hline & & 11 & Skull and neck fractures \\
\hline & & 12 & $\begin{array}{l}\text { Neck neurological } \\
\text { injuries }\end{array}$ \\
\hline & & 17 & Jaw sprains \\
\hline & & 18 & Neck muscle strains \\
\hline & & 19 & Neck sprains \\
\hline \multirow[t]{11}{*}{2} & $\begin{array}{l}\text { Shoulder/arm/ } \\
\text { elbow }\end{array}$ & 21 & $\begin{array}{l}\text { Shoulder sprains and } \\
\text { dislocations }\end{array}$ \\
\hline & & 22 & $\begin{array}{l}\text { A-C (acromioclavicular) } \\
\text { joint }\end{array}$ \\
\hline & & 23 & Fractured clavicles \\
\hline & & 24 & Shoulder tendon injuries \\
\hline & & 25 & $\begin{array}{l}\text { Other arm and elbow } \\
\text { fractures }\end{array}$ \\
\hline & & 26 & $\begin{array}{l}\text { Shoulder and arm stress } \\
\text { fractures }\end{array}$ \\
\hline & & 29 & $\begin{array}{l}\text { Shoulder and arm } \\
\text { neurovascular }\end{array}$ \\
\hline & & 30 & $\begin{array}{l}\text { Upper arm muscle } \\
\text { strains }\end{array}$ \\
\hline & & 32 & $\begin{array}{l}\text { Shoulder and arm soft } \\
\text { tissue trauma }\end{array}$ \\
\hline & & 33 & $\begin{array}{l}\text { Elbow sprains or joint } \\
\text { injuries }\end{array}$ \\
\hline & & 34 & Elbow tendon injuries \\
\hline \multirow[t]{8}{*}{3} & Forearm/wrist/ & 40 & Forearm fractures \\
\hline & hand & 41 & Scaphoid fractures \\
\hline & & 44 & $\begin{array}{l}\text { Other wrist and hand } \\
\text { fractures }\end{array}$ \\
\hline & & 45 & $\begin{array}{l}\text { Forearm and hand stress } \\
\text { fractures }\end{array}$ \\
\hline & & 46 & $\begin{array}{l}\text { Forearm and hand soft } \\
\text { tissue trauma }\end{array}$ \\
\hline & & 47 & $\begin{array}{l}\text { Forearm and hand } \\
\text { neurovascular }\end{array}$ \\
\hline & & 48 & Hand tendon injuries \\
\hline & & 49 & $\begin{array}{l}\text { Wrist and hand sprains } \\
\text { and dislocations }\end{array}$ \\
\hline \multirow[t]{7}{*}{4} & Trunk/back/ & 51 & Rib fractures \\
\hline & buttock & 52 & $\begin{array}{l}\text { Rib and costochondral } \\
\text { bruising }\end{array}$ \\
\hline & & 53 & S/C joint sprains \\
\hline & & 54 & $\begin{array}{l}\text { Abdominal and thoracic } \\
\text { organ damage }\end{array}$ \\
\hline & & 55 & $\begin{array}{l}\text { Lumbar and thoracic } \\
\text { fractures }\end{array}$ \\
\hline & & 56 & Rib stress fractures \\
\hline & & 57 & Pneumothorax \\
\hline
\end{tabular}


Table I (Continued)

\begin{tabular}{|c|c|c|c|}
\hline Region ID & Region & Injcat ID & Injury category \\
\hline & & 59 & $\begin{array}{l}\text { Lumbar and thoracic } \\
\text { soft tissue trauma }\end{array}$ \\
\hline & & 60 & Buttock injuries \\
\hline & & 61 & $\begin{array}{l}\text { Lumbar and thoracic } \\
\text { sprains }\end{array}$ \\
\hline & & 62 & Lumbar stress fractures \\
\hline & & 63 & Trunk muscle strains \\
\hline \multirow[t]{9}{*}{5} & Groin/hip/thigh & 72 & Hip joint injuries \\
\hline & & 73 & $\begin{array}{l}\text { Groin and thigh stress } \\
\text { fractures }\end{array}$ \\
\hline & & 74 & $\begin{array}{l}\text { Hip and groin } \\
\text { contusions }\end{array}$ \\
\hline & & 75 & Groin strain injuries \\
\hline & & 76 & $\begin{array}{l}\text { Pelvic and thigh } \\
\text { fractures }\end{array}$ \\
\hline & & 77 & $\begin{array}{l}\text { Groin and thigh } \\
\text { neurovascular }\end{array}$ \\
\hline & & 81 & Hamstring strains \\
\hline & & 82 & Quadriceps strains \\
\hline & & 83 & Thigh contusions \\
\hline \multirow[t]{10}{*}{6} & Knee & 91 & $\begin{array}{l}\text { Knee - ACL (anterior } \\
\text { cruciate ligament) }\end{array}$ \\
\hline & & 92 & $\begin{array}{l}\text { Knee - MCL (medial } \\
\text { ligament) }\end{array}$ \\
\hline & & 93 & $\begin{array}{l}\text { Knee - PCL (posterior } \\
\text { cruciate ligament) }\end{array}$ \\
\hline & & 94 & Knee cartilage injuries \\
\hline & & 95 & $\begin{array}{l}\text { Knee and patellar } \\
\text { tendon injuries }\end{array}$ \\
\hline & & 96 & Other knee sprains \\
\hline & & 97 & Patella instability \\
\hline & & 98 & Patella stress fractures \\
\hline & & 99 & $\begin{array}{l}\text { Knee and patella } \\
\text { fractures }\end{array}$ \\
\hline & & 100 & Knee contusions \\
\hline \multirow[t]{12}{*}{7} & Lower leg/foot/ & 101 & Leg fractures \\
\hline & ankle & 102 & Leg stress fractures \\
\hline & & 103 & Calf strains \\
\hline & & 104 & $\begin{array}{l}\text { Leg and foot soft tissue } \\
\text { trauma }\end{array}$ \\
\hline & & 105 & Shin soreness \\
\hline & & 106 & Achilles tendon \\
\hline & & 107 & $\begin{array}{l}\text { Ankle sprains and joint } \\
\text { injuries }\end{array}$ \\
\hline & & 111 & Foot bone fractures \\
\hline & & 112 & Foot stress fractures \\
\hline & & 113 & $\begin{array}{l}\text { Foot and ankle } \\
\text { neurovascular }\end{array}$ \\
\hline & & 118 & $\begin{array}{l}\text { Other shin and foot } \\
\text { stress injuries }\end{array}$ \\
\hline & & 119 & Foot sprains \\
\hline \multirow[t]{2}{*}{8} & Medical illness & 121 & Illness, general \\
\hline & & 122 & $\begin{array}{l}\text { Environment-related } \\
\text { illness }\end{array}$ \\
\hline
\end{tabular}

Table 2 Suggested specific injury categories for Australian football and cricket, further merged from the categories in Table I

\begin{tabular}{|c|c|c|}
\hline Injcat ID & $\begin{array}{l}\text { Australian football } \\
\text { category }\end{array}$ & Cricket category \\
\hline I & $\begin{array}{l}\text { Other head and neck } \\
\text { injuries }\end{array}$ & $\begin{array}{l}\text { Other head and neck } \\
\text { injuries }\end{array}$ \\
\hline 3 & $\begin{array}{l}\text { Other head and neck } \\
\text { injuries }\end{array}$ & $\begin{array}{l}\text { Other head and neck } \\
\text { injuries }\end{array}$ \\
\hline 5 & Concussion & $\begin{array}{l}\text { Other head and neck } \\
\text { injuries }\end{array}$ \\
\hline 6 & Facial fractures & Facial fractures \\
\hline 9 & $\begin{array}{l}\text { Other head and neck } \\
\text { injuries }\end{array}$ & $\begin{array}{l}\text { Other head and neck } \\
\text { injuries }\end{array}$ \\
\hline II & $\begin{array}{l}\text { Other head and neck } \\
\text { injuries }\end{array}$ & $\begin{array}{l}\text { Other head and neck } \\
\text { injuries }\end{array}$ \\
\hline 12 & $\begin{array}{l}\text { Other head and neck } \\
\text { injuries }\end{array}$ & $\begin{array}{l}\text { Other head and neck } \\
\text { injuries }\end{array}$ \\
\hline 17 & $\begin{array}{l}\text { Other head and neck } \\
\text { injuries }\end{array}$ & $\begin{array}{l}\text { Other head and neck } \\
\text { injuries }\end{array}$ \\
\hline 18 & $\begin{array}{l}\text { Other head and neck } \\
\text { injuries }\end{array}$ & $\begin{array}{l}\text { Other head and neck } \\
\text { injuries }\end{array}$ \\
\hline 19 & Neck sprains & $\begin{array}{l}\text { Other head and neck } \\
\text { injuries }\end{array}$ \\
\hline 21 & $\begin{array}{l}\text { Shoulder sprains and } \\
\text { dislocations }\end{array}$ & Other shoulder injuries \\
\hline 22 & $\mathrm{~A} / \mathrm{C}$ joint injuries & Other shoulder injuries \\
\hline 23 & Fractured clavicles & $\begin{array}{l}\text { Shoulder/arm/elbow } \\
\text { fractures }\end{array}$ \\
\hline 24 & $\begin{array}{l}\text { Other shoulder/arm/ } \\
\text { elbow injuries }\end{array}$ & $\begin{array}{l}\text { Shoulder/elbow tendon } \\
\text { injuries }\end{array}$ \\
\hline 25 & $\begin{array}{l}\text { Other shoulder/arm/ } \\
\text { elbow injuries }\end{array}$ & $\begin{array}{l}\text { Shoulder/arm/elbow } \\
\text { fractures }\end{array}$ \\
\hline 26 & $\begin{array}{l}\text { Other shoulder/arm/ } \\
\text { elbow injuries }\end{array}$ & $\begin{array}{l}\text { Shoulder/arm/elbow } \\
\text { fractures }\end{array}$ \\
\hline 29 & $\begin{array}{l}\text { Other shoulder/arm/ } \\
\text { elbow injuries }\end{array}$ & Other shoulder injuries \\
\hline 30 & $\begin{array}{l}\text { Other shoulder/arm/ } \\
\text { elbow injuries }\end{array}$ & $\begin{array}{l}\text { Other shoulder/arm/ } \\
\text { elbow injuries }\end{array}$ \\
\hline 32 & $\begin{array}{l}\text { Other shoulder/arm/ } \\
\text { elbow injuries }\end{array}$ & Other shoulder injuries \\
\hline 33 & $\begin{array}{l}\text { Elbow sprains or joint } \\
\text { injuries }\end{array}$ & $\begin{array}{l}\text { Elbow sprains or joint } \\
\text { injuries }\end{array}$ \\
\hline 34 & $\begin{array}{l}\text { Other shoulder/arm/ } \\
\text { elbow injuries }\end{array}$ & $\begin{array}{l}\text { Shoulder/elbow tendon } \\
\text { injuries }\end{array}$ \\
\hline 40 & $\begin{array}{l}\text { Forearm/wrist/hand } \\
\text { fractures }\end{array}$ & $\begin{array}{l}\text { Forearm/wrist/hand } \\
\text { fractures }\end{array}$ \\
\hline $4 I$ & $\begin{array}{l}\text { Forearm/wrist/hand } \\
\text { fractures }\end{array}$ & $\begin{array}{l}\text { Forearm/wrist/hand } \\
\text { fractures }\end{array}$ \\
\hline 44 & $\begin{array}{l}\text { Forearm/wrist/hand } \\
\text { fractures }\end{array}$ & $\begin{array}{l}\text { Forearm/wrist/hand } \\
\text { fractures }\end{array}$ \\
\hline 45 & $\begin{array}{l}\text { Forearm/wrist/hand } \\
\text { fractures }\end{array}$ & $\begin{array}{l}\text { Forearm/wrist/hand } \\
\text { fractures }\end{array}$ \\
\hline 46 & $\begin{array}{l}\text { Other hand/forearm/ } \\
\text { wrist injuries }\end{array}$ & Other wrist/hand injuries \\
\hline 47 & $\begin{array}{l}\text { Other hand/forearm/ } \\
\text { wrist injuries }\end{array}$ & Other wrist/hand injuries \\
\hline 48 & $\begin{array}{l}\text { Other hand/forearm/ } \\
\text { wrist injuries }\end{array}$ & Other wrist/hand injuries \\
\hline
\end{tabular}


Table 2 (Continued)

\begin{tabular}{|c|c|c|}
\hline Injcat ID & $\begin{array}{l}\text { Australian football } \\
\text { category }\end{array}$ & Cricket category \\
\hline 49 & $\begin{array}{l}\text { Other hand/forearm/ } \\
\text { wrist injuries }\end{array}$ & Other wrist/hand injuries \\
\hline 51 & $\begin{array}{l}\text { Rib and chest wall } \\
\text { injuries }\end{array}$ & $\begin{array}{l}\text { Other buttock/back/trunk } \\
\text { injuries }\end{array}$ \\
\hline 52 & $\begin{array}{l}\text { Rib and chest wall } \\
\text { injuries }\end{array}$ & $\begin{array}{l}\text { Other buttock/back/trunk } \\
\text { injuries }\end{array}$ \\
\hline 53 & $\begin{array}{l}\text { Rib and chest wall } \\
\text { injuries }\end{array}$ & $\begin{array}{l}\text { Other buttock/back/trunk } \\
\text { injuries }\end{array}$ \\
\hline 54 & $\begin{array}{l}\text { Other buttock/back/ } \\
\text { trunk injuries }\end{array}$ & $\begin{array}{l}\text { Other buttock/back/trunk } \\
\text { injuries }\end{array}$ \\
\hline 55 & $\begin{array}{l}\text { Lumbar and thoracic } \\
\text { spine injuries }\end{array}$ & $\begin{array}{l}\text { Other buttock/back/trunk } \\
\text { injuries }\end{array}$ \\
\hline 56 & Rib and chest wall injuries & Side and abdominal strains \\
\hline 57 & Rib and chest wall injuries & $\begin{array}{l}\text { Other buttock/back/trunk } \\
\text { injuries }\end{array}$ \\
\hline 59 & $\begin{array}{l}\text { Lumbar and thoracic } \\
\text { spine injuries }\end{array}$ & $\begin{array}{l}\text { Other buttock/back/trunk } \\
\text { injuries }\end{array}$ \\
\hline 60 & $\begin{array}{l}\text { Other buttock/back/ } \\
\text { trunk injuries }\end{array}$ & $\begin{array}{l}\text { Other buttock/back/trunk } \\
\text { injuries }\end{array}$ \\
\hline 61 & $\begin{array}{l}\text { Lumbar and thoracic } \\
\text { spine injuries }\end{array}$ & $\begin{array}{l}\text { Other buttock/back/trunk } \\
\text { injuries }\end{array}$ \\
\hline 62 & $\begin{array}{l}\text { Lumbar and thoracic } \\
\text { spine injuries }\end{array}$ & Lumbar stress fractures \\
\hline 63 & $\begin{array}{l}\text { Other buttock/back/ } \\
\text { trunk injuries }\end{array}$ & Side and abdominal strains \\
\hline 72 & $\begin{array}{l}\text { Other hip/groin/thigh } \\
\text { injuries }\end{array}$ & $\begin{array}{l}\text { Other hip/groin/thigh } \\
\text { injuries }\end{array}$ \\
\hline 73 & $\begin{array}{l}\text { Other hip/groin/thigh } \\
\text { injuries }\end{array}$ & $\begin{array}{l}\text { Other hip/groin/thigh } \\
\text { injuries }\end{array}$ \\
\hline 74 & Thigh and hip contusions & $\begin{array}{l}\text { Other hip/groin/thigh } \\
\text { injuries }\end{array}$ \\
\hline 75 & $\begin{array}{l}\text { Groin strains and osteitis } \\
\text { pubis }\end{array}$ & $\begin{array}{l}\text { Groin strains and osteitis } \\
\text { pubis }\end{array}$ \\
\hline 76 & $\begin{array}{l}\text { Other hip/groin/thigh } \\
\text { injuries }\end{array}$ & $\begin{array}{l}\text { Other hip/groin/thigh } \\
\text { injuries }\end{array}$ \\
\hline 77 & $\begin{array}{l}\text { Groin strains and osteitis } \\
\text { pubis }\end{array}$ & $\begin{array}{l}\text { Other hip/groin/thigh } \\
\text { injuries }\end{array}$ \\
\hline 81 & Hamstring strains & Hamstring strains \\
\hline 82 & Quadriceps strains & Quadriceps strains \\
\hline 83 & Thigh and hip contusions & $\begin{array}{l}\text { Other hip/groin/thigh } \\
\text { injuries }\end{array}$ \\
\hline 91 & Knee ACL & Knee ligament injuries \\
\hline 92 & Knee MCL & Knee ligament injuries \\
\hline 93 & Knee PCL & Knee ligament injuries \\
\hline 94 & Knee cartilage & Knee cartilage \\
\hline 95 & $\begin{array}{l}\text { Knee and patella tendon } \\
\text { injuries }\end{array}$ & Other knee injuries \\
\hline 96 & Other knee injuries & Other knee injuries \\
\hline 97 & Patella injuries & Other knee injuries \\
\hline 98 & Patella injuries & Other knee injuries \\
\hline 99 & Other knee injuries & Other knee injuries \\
\hline 100 & Other knee injuries & Other knee injuries \\
\hline 101 & Leg and foot fractures & $\begin{array}{l}\text { Other leg/foot/ankle } \\
\text { injuries }\end{array}$ \\
\hline
\end{tabular}

Table 2 (Continued)

\begin{tabular}{|c|c|c|}
\hline Injcat ID & $\begin{array}{l}\text { Australian football } \\
\text { category }\end{array}$ & Cricket category \\
\hline 102 & $\begin{array}{l}\text { Lower leg/foot stress } \\
\text { fractures }\end{array}$ & $\begin{array}{l}\text { Lower leg/foot stress } \\
\text { fractures }\end{array}$ \\
\hline 103 & Calf strains & Calf strains \\
\hline 104 & $\begin{array}{l}\text { Other leg/foot/ankle } \\
\text { injuries }\end{array}$ & $\begin{array}{l}\text { Other leg/foot/ankle } \\
\text { injuries }\end{array}$ \\
\hline 105 & $\begin{array}{l}\text { Other leg/foot/ankle } \\
\text { injuries }\end{array}$ & $\begin{array}{l}\text { Other leg/foot/ankle } \\
\text { injuries }\end{array}$ \\
\hline 106 & Achilles tendon injuries & $\begin{array}{l}\text { Other leg/foot/ankle } \\
\text { injuries }\end{array}$ \\
\hline 107 & $\begin{array}{l}\text { Ankle sprains or joint } \\
\text { injuries }\end{array}$ & $\begin{array}{l}\text { Ankle sprains or joint } \\
\text { injuries }\end{array}$ \\
\hline III & Leg and foot fractures & $\begin{array}{l}\text { Other leg/foot/ankle } \\
\text { injuries }\end{array}$ \\
\hline 112 & $\begin{array}{l}\text { Lower leg/foot stress } \\
\text { fractures }\end{array}$ & $\begin{array}{l}\text { Lower leg/foot stress } \\
\text { fractures }\end{array}$ \\
\hline 113 & $\begin{array}{l}\text { Other leg/foot/ankle } \\
\text { injuries }\end{array}$ & $\begin{array}{l}\text { Other leg/foot/ankle } \\
\text { injuries }\end{array}$ \\
\hline 118 & $\begin{array}{l}\text { Other leg/foot/ankle } \\
\text { injuries }\end{array}$ & $\begin{array}{l}\text { Other leg/foot/ankle } \\
\text { injuries }\end{array}$ \\
\hline 119 & $\begin{array}{l}\text { Other leg/foot/ankle } \\
\text { injuries }\end{array}$ & $\begin{array}{l}\text { Other leg/foot/ankle } \\
\text { injuries }\end{array}$ \\
\hline$|2|$ & Medical illnesses & Medical illnesses \\
\hline 122 & Medical illnesses & $\begin{array}{l}\text { Environment-related } \\
\text { illness }\end{array}$ \\
\hline
\end{tabular}

Table 3 Example specific injury categories for football (soccer) and rugby union, further merged from the categories in Table I

\begin{tabular}{lll}
\hline Injcat ID & Soccer category & Rugby union category \\
\hline I & $\begin{array}{l}\text { Other head and neck } \\
\text { injuries }\end{array}$ & $\begin{array}{l}\text { Other head and neck } \\
\text { injuries }\end{array}$ \\
3 & $\begin{array}{l}\text { Other head and neck } \\
\text { injuries }\end{array}$ & $\begin{array}{l}\text { Other head and neck } \\
\text { injuries }\end{array}$ \\
5 & Concussion & Concussion \\
6 & Facial fractures & Facial fractures \\
9 & Other head and neck & Other head and neck \\
& injuries & injuries \\
11 & Other head and neck & Other head and neck \\
& injuries & injuries \\
12 & Other head and neck injuries & Neck stingers/burners \\
17 & Other head and neck & Other head and neck \\
& injuries & injuries \\
18 & Other head and neck & Other head and neck \\
& injuries & injuries \\
19 & Other head and neck injuries & Neck sprains \\
21 & Shoulder sprains and & Shoulder sprains and \\
dislocations & dislocations \\
22 & A/C joint injuries & A/C joint injuries \\
23 & Fractured clavicles & Fractured clavicles \\
24 & Other shoulder/arm/ & Other shoulder/arm/ \\
& elbow injuries & elbow injuries \\
25 & Other shoulder/arm/ & Other shoulder/arm/ \\
& elbow injuries & elbow injuries \\
\hline & &
\end{tabular}


Table 3 (Continued)

\begin{tabular}{|c|c|c|c|c|c|}
\hline Injcat ID & Soccer category & Rugby union category & Injcat ID & Soccer category & Rugby union category \\
\hline 26 & $\begin{array}{l}\text { Other shoulder/arm/ } \\
\text { elbow injuries }\end{array}$ & $\begin{array}{l}\text { Other shoulder/arm/ } \\
\text { elbow injuries }\end{array}$ & 77 & $\begin{array}{l}\text { Groin strains and osteitis } \\
\text { pubis }\end{array}$ & $\begin{array}{l}\text { Groin strains and osteitis } \\
\text { pubis }\end{array}$ \\
\hline 29 & $\begin{array}{l}\text { Other shoulder/arm/ } \\
\text { elbow injuries }\end{array}$ & $\begin{array}{l}\text { Other shoulder/arm/ } \\
\text { elbow injuries }\end{array}$ & $\begin{array}{l}81 \\
82\end{array}$ & $\begin{array}{l}\text { Hamstring strains } \\
\text { Quadriceps strains }\end{array}$ & $\begin{array}{l}\text { Hamstring strains } \\
\text { Quadriceps strains }\end{array}$ \\
\hline 30 & $\begin{array}{l}\text { Other shoulder/arm/ } \\
\text { elbow injuries }\end{array}$ & $\begin{array}{l}\text { Other shoulder/arm/ } \\
\text { elbow injuries }\end{array}$ & $\begin{array}{l}83 \\
91\end{array}$ & $\begin{array}{l}\text { Thigh and hip contusions } \\
\text { Knee } A C L\end{array}$ & $\begin{array}{l}\text { Thigh and hip contusions } \\
\text { Knee ACL }\end{array}$ \\
\hline 32 & $\begin{array}{l}\text { Other shoulder/arm/ } \\
\text { elbow injuries }\end{array}$ & $\begin{array}{l}\text { Other shoulder/arm/ } \\
\text { elbow injuries }\end{array}$ & $\begin{array}{l}92 \\
93\end{array}$ & $\begin{array}{l}\text { Knee MCL } \\
\text { Knee PCL }\end{array}$ & $\begin{array}{l}\text { Knee MCL } \\
\text { Knee PCL }\end{array}$ \\
\hline 33 & $\begin{array}{l}\text { Other shoulder/arm/ } \\
\text { elbow injuries }\end{array}$ & $\begin{array}{l}\text { Elbow sprains or joint } \\
\text { injuries }\end{array}$ & $\begin{array}{l}94 \\
95\end{array}$ & $\begin{array}{l}\text { Knee cartilage } \\
\text { Knee and patella tendon }\end{array}$ & $\begin{array}{l}\text { Knee cartilage } \\
\text { Knee and patella tendon }\end{array}$ \\
\hline 34 & $\begin{array}{l}\text { Other shoulder/arm/ } \\
\text { elbow injuries }\end{array}$ & $\begin{array}{l}\text { Other shoulder/arm/ } \\
\text { elbow injuries }\end{array}$ & 96 & $\begin{array}{l}\text { injuries } \\
\text { Other knee injuries }\end{array}$ & $\begin{array}{l}\text { injuries } \\
\text { Other knee injuries }\end{array}$ \\
\hline 40 & $\begin{array}{l}\text { Forearm/wrist/hand } \\
\text { injuries }\end{array}$ & $\begin{array}{l}\text { Forearm/wrist/hand } \\
\text { fractures }\end{array}$ & $\begin{array}{l}97 \\
98\end{array}$ & $\begin{array}{l}\text { Patella injuries } \\
\text { Patella injuries }\end{array}$ & $\begin{array}{l}\text { Patella injuries } \\
\text { Patella injuries }\end{array}$ \\
\hline $4 \mathrm{I}$ & $\begin{array}{l}\text { Forearm/wrist/hand } \\
\text { injuries }\end{array}$ & $\begin{array}{l}\text { Forearm/wrist/hand } \\
\text { fractures }\end{array}$ & $\begin{array}{l}99 \\
100\end{array}$ & $\begin{array}{l}\text { Other knee injuries } \\
\text { Other knee injuries }\end{array}$ & $\begin{array}{l}\text { Other knee injuries } \\
\text { Other knee injuries }\end{array}$ \\
\hline 44 & $\begin{array}{l}\text { Forearm/wrist/hand } \\
\text { injuries }\end{array}$ & $\begin{array}{l}\text { Forearm/wrist/hand } \\
\text { fractures }\end{array}$ & $\begin{array}{l}101 \\
102\end{array}$ & $\begin{array}{l}\text { Leg and foot fractures } \\
\text { Lower leg/foot stress }\end{array}$ & $\begin{array}{l}\text { Leg and foot fractures } \\
\text { Lower leg/foot stress }\end{array}$ \\
\hline 45 & $\begin{array}{l}\text { Forearm/wrist/hand } \\
\text { injuries }\end{array}$ & $\begin{array}{l}\text { Forearm/wrist/hand } \\
\text { fractures }\end{array}$ & 103 & $\begin{array}{l}\text { fractures } \\
\text { Calf strains }\end{array}$ & $\begin{array}{l}\text { fractures } \\
\text { Calf strains }\end{array}$ \\
\hline 46 & $\begin{array}{l}\text { Forearm/wrist/hand } \\
\text { injuries }\end{array}$ & $\begin{array}{l}\text { Other hand/forearm/ } \\
\text { wrist injuries }\end{array}$ & 104 & $\begin{array}{l}\text { Other leg/foot/ankle } \\
\text { injuries }\end{array}$ & $\begin{array}{l}\text { Other leg/foot/ankle } \\
\text { injuries }\end{array}$ \\
\hline 47 & $\begin{array}{l}\text { Forearm/wrist/hand } \\
\text { injuries }\end{array}$ & $\begin{array}{l}\text { Other hand/forearm/ } \\
\text { wrist injuries }\end{array}$ & 105 & $\begin{array}{l}\text { Other leg/foot/ankle } \\
\text { injuries }\end{array}$ & $\begin{array}{l}\text { Other leg/foot/ankle } \\
\text { injuries }\end{array}$ \\
\hline 48 & $\begin{array}{l}\text { Forearm/wrist/hand } \\
\text { injuries }\end{array}$ & $\begin{array}{l}\text { Other hand/forearm/ } \\
\text { wrist injuries }\end{array}$ & $\begin{array}{l}106 \\
107\end{array}$ & $\begin{array}{l}\text { Achilles tendon injuries } \\
\text { Ankle sprains or joint }\end{array}$ & $\begin{array}{l}\text { Achilles tendon injuries } \\
\text { Ankle sprains or joint }\end{array}$ \\
\hline 49 & $\begin{array}{l}\text { Forearm/wrist/hand } \\
\text { injuries }\end{array}$ & $\begin{array}{l}\text { Other hand/forearm/ } \\
\text { wrist injuries }\end{array}$ & III & $\begin{array}{l}\text { injuries } \\
\text { Leg and foot fractures }\end{array}$ & $\begin{array}{l}\text { injuries } \\
\text { Leg and foot fractures }\end{array}$ \\
\hline $\begin{array}{l}51 \\
52\end{array}$ & $\begin{array}{l}\text { Rib and chest wall injuries } \\
\text { Rib and chest wall injuries }\end{array}$ & $\begin{array}{l}\text { Rib and chest wall injuries } \\
\text { Rib and chest wall injuries }\end{array}$ & 112 & $\begin{array}{l}\text { Lower leg/foot stress } \\
\text { fractures }\end{array}$ & $\begin{array}{l}\text { Lower leg/foot stress } \\
\text { fractures }\end{array}$ \\
\hline 53 & Rib and chest wall injuries & Rib and chest wall injuries & 113 & Other leg/foot/ankle & Other leg/foot/ankle \\
\hline 54 & Other buttock/back/trunk & Other buttock/back/trunk & & injuries & injuries \\
\hline & injuries & injuries & 118 & Other leg/foot/ankle & Other leg/foot/ankle \\
\hline 55 & $\begin{array}{l}\text { Lumbar and thoracic spine } \\
\text { injuries }\end{array}$ & $\begin{array}{l}\text { Lumbar and thoracic } \\
\text { spine injuries }\end{array}$ & 119 & $\begin{array}{l}\text { injuries } \\
\text { Other leg/foot/ankle }\end{array}$ & $\begin{array}{l}\text { injuries } \\
\text { Other leg/foot/ankle }\end{array}$ \\
\hline 56 & Rib and chest wall injuries & Rib and chest wall injuries & & injuries & injuries \\
\hline 57 & Rib and chest wall injuries & Rib and chest wall injuries & $|2|$ & Medical illnesses & Medical illnesses \\
\hline 59 & Lumbar and thoracic & Lumbar and thoracic & 122 & Medical illnesses & Medical illnesses \\
\hline
\end{tabular}

spine injuries

trunk injuries

Lumbar and thoracic spine injuries

Lumbar and thoracic spine injuries

Other buttock/back/

trunk injuries

Other hip/groin/thigh

\section{injuries}

Other hip/groin/thigh

injuries

Groin strains and osteitis

pubis

Other hip/groin/thigh injuries
Other buttock/back/

Thigh and hip contusions

\section{spine injuries}

Other buttock/back/

trunk injuries

Lumbar and thoracic

spine injuries

Lumbar and thoracic

spine injuries

Other buttock/back/

trunk injuries

Other hip/groin/thigh

injuries

Other hip/groin/thigh

injuries

Thigh and hip contusions

Groin strains and osteitis

pubis

Other hip/groin/thigh

injuries
Table 3 (Continued)

Some sports/users may prefer not to combine body parts and injury types in the same table. For example, in the soccer ${ }^{1}$ and tennis ${ }^{32,33}$ consensus statements the groups recommended tabulating separately by body area and then by injury type. It is easier (but still not straightforward) to determine the boundary between categories when choosing a 'body part' or 'injury type' list, compared to a more global injury category list. For example, should groin/hip/thigh be a body part category or separated into groin/hip and thigh? And if it is separated, are all adductor muscle strains to be included in the groin section, or does it depend on whether they are proximal (groin) or distal (thigh)? It is useful to be able to read, say, the rate of 'muscle strains' from a surveillance system and also the rate of 'thigh injuries'. Where 
the consensus statements currently recommend tabulating separately by body part and by injury type then obviously this format is recommended.

However, many readers of reports will specifically want to know the rate of common injuries, such as 'hamstring injuries' for example. This rate is not clear if only categorization is made by body part and then by injury type. If a hybrid table is formed (as has been attempted in Tables 2 and 3) there needs to be agreement on which injuries warrant their own separate category (balanced against the need to keep the table a reasonable size). Depending on how common hamstring injuries are in a sport, they can form their own separate injury category or can be combined with quadriceps strains (to form a category of 'hamstring and thigh muscle strains') or with groin and quadriceps muscle strains (to form a category of 'upper leg muscle strains'). Because of the multiple approaches of tabulating categories, it is suggested that each sport includes this issue as part of future consensus statements or consensus statement updates.

New lists of injury codes for versions 9 (three digit codes) and 10.1 (four digit codes) are available from http:// injuryupdate.com.au/research/OSICS.htm. OSICS version 10 has recently been translated into Spanish (http://www.apunts. org/ficheros/apunts/videos/ocsis10-es.xls) and Catalan, ${ }^{35}$ with further versions possible in other languages.

\section{Discussion}

This paper presents for the first time OSICS version 9, which is essentially the first major modification of OSICS 8, retaining the three code system. It is ideal for use in an injury management system where easy and simple coding is preferred. This is most likely to be the case where the main purpose of coding is to assist grouping into larger injury categories for presentation in reports or scientific papers. Where the aims of an injury surveillance system also involve an archiving function, to be able to retrieve records with greater specificity, OSICS version 10 is preferred. This study also presents version 10.1, which is very similar to the original version 10 but contains a few minor modifications (hence does not qualify as a major rewrite, which may one day be done with a version 11).

One of the major advantages of OSICS is the fact that the system is available worldwide for free use. This fact alone is a good explanation for the popularity and uptake of the system. However, the fact that no income is earned by the system means that updates and product support are below an ideal level. Most users would probably prefer suboptimal support and an ongoing free system compared to a product licence with a greater level of support for queries and system implementation.
Ideally, in the future, a major update of OSICS (say to version 11) would involve a formal consultative process in a similar fashion to updates of the ICD. Part of the process could include, for example, multiple expert and novice users attempting to code a long list of provided text diagnoses. Where there was broad agreement amongst the users, no changes to codes would be deemed necessary. Where users were in disagreement or unable to agree on a suitable OSICS code, a new or modified code could be established for the new version. Such a process would be time- and resourceconsuming and would require funding. If a private body provided the funding for such a process, it may require assignment of copyright in the new system in return. This would obviously improve the system, but potentially at a cost of the new version being freely available for use.

It is important to note that OSICS is not a comprehensive injury surveillance system itself, only a system for coding major diagnoses. Diagnosis and injury code are important fields in a sports injury database or injury surveillance system. ${ }^{36}$ Other data which are also relevant are injury side, injury mechanism (eg, noncontact vs contact), date of onset, date of return (severity), activity of onset (eg, match play, training, insidious onset) and exposure time. Another dilemma which users and coordinators of injury surveillance systems must address is whether to allow multiple injury codes/diagnoses for single events. For example, a valgus mechanism knee injury is a single event but may be associated with a combination of injuries (such as medial collateral ligament (MCL) + anterior cruciate ligament (ACL) sprains). This could be coded as a single injury, with deference given to the more significant of the two diagnoses (ie, ACL injury is of more significance than MCL injury). Alternatively it could be coded as two different injuries with the same mechanism and date/time of onset. It is also possible in an injury surveillance system for a single injury event to contain multiple injury codes as part of the one 'injury' (ie, combined ACL/MCL diagnosis).

Because there are so many similar dilemmas when conducting injury surveillance, it is recommended that for sports where many different groups are undertaking injury surveillance that consensus papers be developed. ${ }^{1-4}$ With respect to use of OSICS, for those consensus groups that decide to recommend it, there would be a lot of benefit in suggesting broad injury categories for tabulation (cricket consensus statement paper ${ }^{3,4,37}$ ). The more common ground there is between various research groups in their methodology, the more valid are comparisons between studies undertaken by different author groups. 


\section{Disclosures}

The authors report no conflicts of interest in this work.

\section{References}

1. Fuller C, Ekstrand J, Junge A, Andersen T, Bahr R, Dvorak J, et al. Consensus statement on injury definitions and data collection procedures in studies of football (soccer) injuries. Br J Sports Med. 2006;40:193-201.

2. Fuller C, Molloy M, Bagate C, Bahr R, Brooks J, Donson H, et al. Consensus statement on injury definitions and data collection procedures for studies of injuries in rugby union. Clin J Sport Med. 2007;17(3): 177-181.

3. Orchard J, Newman D, Stretch R, Frost W, Mansingh A, Leipus A. Methods for injury surveillance in international cricket. Br J Sports Med. 2005;39(4):E22.

4. Orchard J, Newman D, Stretch R, Frost W, Mansingh A, Leipus A. Methods for injury surveillance in international cricket. J Sci Med Sport. 2005;8(1):1-14.

5. van Mechelen W, Hlobil H, Kemper H. Incidence, Severity, Aetiology and Prevention of Sports Injuries: A Review of Concepts. Sports Med. 1992;14(2):82-99.

6. Finch C. A new framework for research leading to sports injury prevention. J Sci Med Sport. 2006;9:3-9.

7. Gianotti S, Hume P. A cost-outcome approach to pre and postimplementation of national sports injury prevention programmes. $J \mathrm{Sci}$ Med Sport. 2007;10(6):436-446.

8. Gianotti S, Marshall S, Hume P, Bunt L. Incidence of anterior cruciate ligament injury and other knee ligament injuries: A national populationbased study. J Sci Med Sport. 2009;12(6):622-627.

9. Quarrie K, Gianotti S, Hopkins W, Hume P. Effect of a nationwide injury prevention programme on serious spinal injuries in New Zealand rugby union: ecological study. BMJ. 2007;334:1150-1153.

10. Torg JS, Vegso J, Sennett B, Das M. The National Football Head and Neck Injury Registry. 14-year report on cervical quadriplegia, 1971 through 1984. JAMA. 1985;254(24):3439-3443.

11. Janda D, Wojtys E, Hankin F, Benedict M. Softball sliding injuries. A prospective study comparing standard and modified bases. JAMA. 1988;259:1848-1850.

12. Orchard J, Seward H. Decreased incidence of knee posterior cruciate ligament injury in Australian Football League after ruck rule change. Br J Sports Med. 2009;43:1026-1030.

13. Rae K, Orchard J. The Orchard Sports Injury Classification System (OSICS) Version 10. Clin J Sport Med. 2007;17(3):201-204.

14. Rae K, Britt H, Orchard J, Finch C. Classifying sports medicine diagnoses: a comparison of the International classification of diseases 10-Australian modification (ICD-10-AM) and the Orchard sports injury classification system (OSICS-8). Br J Sports Med. 2005;39:907-911.

15. Hammond L, Lilley J, Ribbans W. Coding sports injury surveillance data: has version 10 of the Orchard Sports Injury Classification System improved the classification of sports medicine diagnoses? Br J Sports Med. 2009;43(7):498-502.

16. Orchard J. Orchard Sports Injury Classification System (OSICS). Sport Health. 1993;11(3):39-41.

17. Orchard J. Orchard Sports Injury Classification System (OSICS). In: Bloomfield J, Fricker P, Fitch K, editors. Science and Medicine in Sport. 2nd ed. Melbourne: Blackwell; 1995 p 674-681.
18. Orchard J, Seward H. AFL Injury Report: Season 2008. Sport Health 2009;27(2):http://www.injuryupdate.com.au/images/research/ AFL_Injury_Report_2008.pdf.

19. Orchard J, Seward H. Epidemiology of injuries in the Australian Football League, seasons 1997-2000. Br J Sports Med. 2002;36:39-45.

20. Orchard J, Verrall G. Groin Injuries in the Australian Football League. ISMJ. 2000;1(1).

21. Seward H, Orchard J, Hazard H, Collinson D. Football Injuries in Australia at the elite level. Med J Aust. 1993;159:298-301.

22. Orchard J. Soft tissue Injuries in the Australian Football League. Koln: Lambert Academic Publishing; 2009.

23. Gabbe B, Finch C, Wajswelner H, Bennell K. Australian football: injury profile at the community level. J Sci Med Sport. 2002;5(2):149-160.

24. Orchard J, James T, Alcott E, Carter S, Farhart P. Injuries in Australian cricket at first class level 1995/96 to 2000/01. Br J Sports Med. 2002;36: 270-275.

25. Orchard J, James T, Portus M. Injuries to elite male cricketers in Australia over a 10-year period. J Sci Med Sport. 2006;9:459-467.

26. Ekstrand J, Hägglund M, Walden M. Injury incidence and injury patterns in professional football - the UEFA injury study. Br J Sports Med. 2010; May 29 [Epub ahead of print].

27. Ekstrand J, Timpka T, Hägglund M. Risk of injury in elite football played on artificial turf versus natural grass: a prospective two-cohort study. Br J Sports Med. 2006;40:975-980.

28. Hägglund M, Walden M, Bahr R, Ekstrand J. Methods for epidemiological study of injuries to professional football players: developing the UEFA model. Br J Sports Med. 2005;39:340-346.

29. McManus A. Validation of an instrument for injury data collection in rugby union. Br J Sports Med. 2000;34:342-347.

30. Kemp S, Brooks J, Fuller C, Hansen K, Lidlow K, Smith A, et al. England Rugby Injury and Training Audit. 2008-2009 season report. London: Rugby Football Union, 2010:http://www.rfu.com/News/2010/ January/News\%20Articles/ /media/Files/2010/ManagingRugby/ Fitness/England\%20Rugby\%20\%20Injury\%20Trainiing\%20Audit $\% 20$ 0809.ashx.

31. Brooks J, Fuller C, Kemp S, Reddin D. Epidemiology of injuries in English professional rugby union: part 1 match injuries. Br J Sports Med. 2005;39(10):757-766.

32. Pluim B, Fuller C, Batt M, Chase L, Hainline B, Miller S, et al. Consensus statement on epidemiological studies of medical conditions in tennis, April 2009. Clin J Sport Med. 2009;19(6):445-450.

33. Pluim B, Fuller C, Batt M, Chase L, Hainline B, Miller S, et al. Consensus statement on epidemiological studies of medical conditions in tennis, April 2009. Br J Sports Med. 2009;43(12):893-897.

34. Junge A, Engebretsen L, Alonso J, Renstrom P, Mountjoy M, Aubry M, et al. Injury surveillance in multi-sport events: the International Olympic Committee approach. Br J Sports Med. 2008;42(6):413-421.

35. Til L, Orchard J, Rae K. The Orchard Sports Injury Classification System (OSICS) version 10. APUNTS. Medicina De L'Esport. 2008;43:109-112.

36. Orchard J, Blood G. How to use databases in sports medicine research. In: MacAuley D, Best T, editors. Evidence-Based Sports Medicine. London: BMJ Books; 2002.

37. Orchard J, Newman D, Stretch R, Frost W, Mansingh A, Leipus A. Methods for injury surveillance in international cricket. South African Journal of Sports Medicine. 2005;17(2):18-28.
Open Access Journal of Sports Medicine

\section{Publish your work in this journal}

Open Access Journal of Sports Medicine is an international, peer-reviewed, open access journal publishing original research, reports, reviews and commentaries on all areas of sports medicine. The manuscript management system is completely online and includes a very quick and fair peer-review system.

\section{Dovepress}

Visit http://www.dovepress.com/testimonials.php to read real quotes from published authors. 